\title{
Dynamic model elaboration for the planetary- precessional gear reducer against vibration
}

\author{
Valeriu Dulgheru* and Iulian Malcoci \\ UTM, Fundamentals Machinery Design Department, MD-2045, str. Studenților 9/8, Rep. Moldova
}

\begin{abstract}
The purpose of modern-day machines in general and gear-box in particular is to carry out needs-based on maintenance, good working conditions and to minimize the repair and other servicing downtimes and disfunctionalities. This increases the overall equipment effectiveness and optimizes the cost. The mechanical condition of a machine or a machine components can be accurately diagnosed from the nature and extend of vibration they generate. The aim is to detect damage as it occurs, allowing scheduled repairs or maintenance to be carried out in time.
\end{abstract}

\section{Vibration generating sources in the planetary-precessional gear}

Precessional transmission is a mechanical system subjected to mechanical oscillations, generating vibrations and noise, and can be divided into the following groups [1]:

a) Dimensional deviations of sliding contact elements;

b) Dimensional deviations in machine parts that make rolling contact;

c) Imbalance, when the center of mass of a rotating or precession element does not coincide with the center of rotation (or precession) [2];

d) Disassembly - deviation from the collinearity of the axles of the electric motor shaft, the precession reducer and the brake, deviation which occurs at normal operating temperature;

e) Vibration resulting from assembly errors [3].

\section{The dynamic model of the planetary precessional reducer elastically bonded against forced vibrations}

If we examine the dynamic model of the planetary precessional reducer, we conclude that the reducer is a rigid system with a vertical plane of symmetry. As with any other gear transmission, precessional planetary transmissions (later on the textPPT) are excited by various disturbing forces.

Figure 1 shows the 2D-simplified planetary $2 \mathrm{~K}-\mathrm{H}$ reducer simplified model. A summary analysis shows that, in general, the reducer consists of two cylinders with diameters D1, D2.

*Corresponding author: iulian.malcoci@bpm.utm.md 


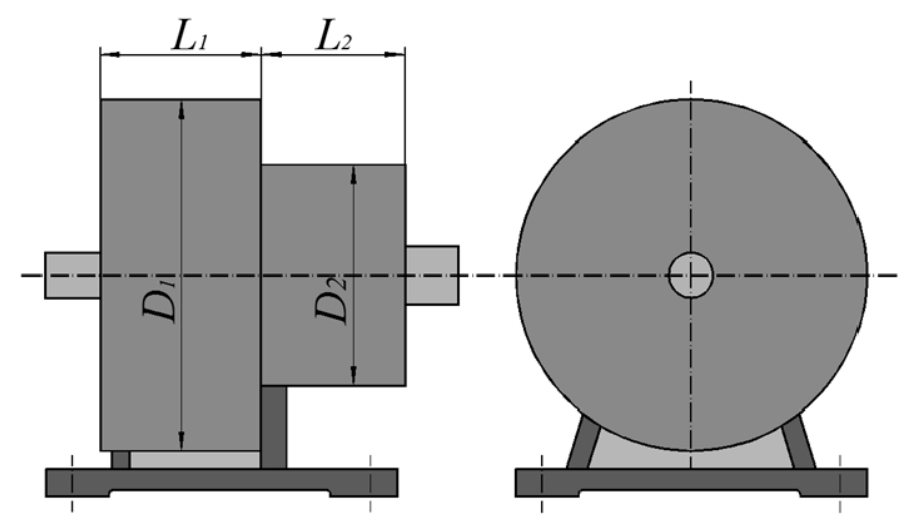

Fig. 1. Simplified 2K-H planetary precessional transmission model.

In this order, the planetary precessional reducer $2 \mathrm{~K}-\mathrm{H}$ can be approximated to a cylinder of diameter $\mathrm{D}_{\mathrm{e}}$ and length $\mathrm{L}_{\mathrm{e}}$, like is shown in Figure 2. [4]

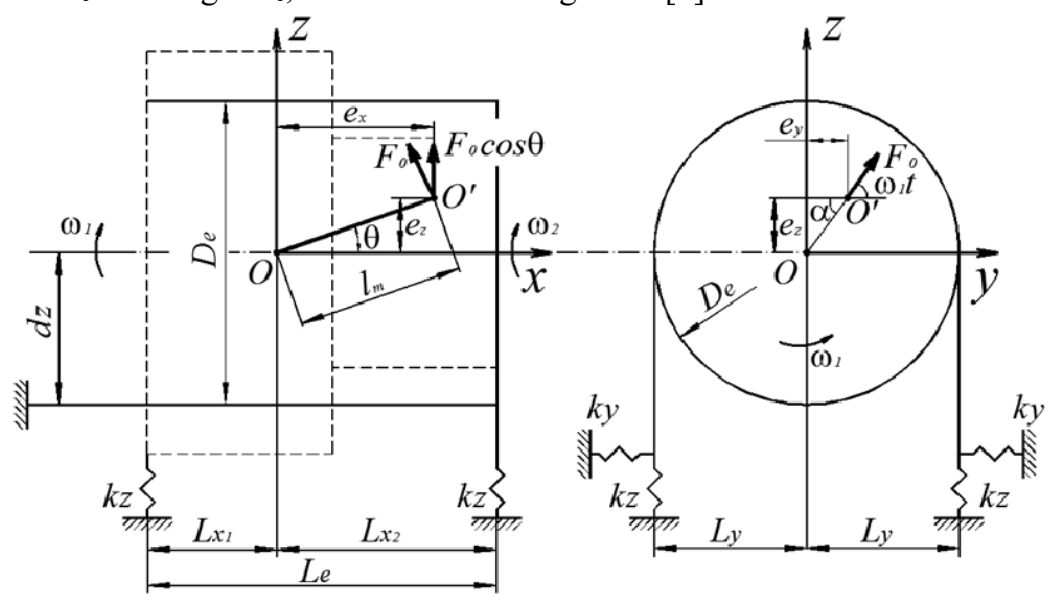

Fig. 2. 2K-H planetary precessional reducer simplified dynamic model.

This simplified model is characterized by the following features:

- the precessional planetary reducer is considered a solid body with two $O x y$ and $O x z$ symmetry planes;

- the resilience is performed in four points, each elastic system being characterized by two orthogonal elastic elements, having the rigidities $k z$ and $k y$ along the axes $O x$ and $O z$;

- the excitation of stationary forced vibrations have an inertial type due to an eccentric mass system (satellite block and crank shaft) with the rotation axis inclined with the angle $\theta$ from the main axle of the transmission ( $O x$ axis) passing through the $O^{\prime}$ point displaced by the center the precession " $O$ " at the distances $e_{y}$ and $e_{z}$ in the $O y z$ plane and $e_{x}$ - in the $O x z$ plane.

$$
\left\{\begin{array}{c}
F_{x}(t)=F_{0} \sin \theta \cos \omega_{1} t=m_{0} r \omega_{1}^{2} \sin \theta ; \\
F_{y}(t)=F_{0} \cos \theta \cos \omega_{1} t \\
F_{z}(t)=F_{0} \cos \theta \sin \omega_{1} t=m_{0} r \omega_{1}^{2} \cos \theta .
\end{array}\right.
$$


The total static momentum of the eccentric masses is $m_{0} r$ and the rotating disturbing force has the application point $O{ }^{\prime}$ offset from the center of precession " $O$ " at the distances $e_{x}, e_{y}$ and $e_{z}$.

The external excitation forces acting on PPT are presented in the expression (1). Correspondingly, the external excitation moments are:

$$
\left\{\begin{array}{c}
M_{0 x}(t)=\left(e_{z} \cos \omega_{1} t-e_{y} \cos \omega_{1} t\right) F_{0} \cos \theta \\
M_{0 y}(t)=F_{0} \cos \theta \cos \omega_{1} t \\
M_{0 z}(t)=F_{0} \cos \theta \sin \omega_{1} t
\end{array}\right.
$$

The moment $M_{0 x}(t)$ can also be written in the form of $M_{0 x}(t)=M_{0} \sin \left(\omega_{1} t-\alpha\right)$ where $M_{0}=F_{0} \cdot y=m_{0} r \omega_{1}^{2} \varphi$ is the amplitude of the excitatory moment, and $\alpha$ is the phase angle of the excitatory force vector:

$$
\alpha=\operatorname{arctg} \frac{e_{z}}{e_{y}}=\operatorname{arctg} \frac{l_{m} \sin \theta}{l_{m} \sin \theta \sin \omega_{1} t}=\operatorname{arctg} \frac{1}{\sin \omega_{1} t} .
$$

In this case summary excitation moment will be:

$$
\begin{aligned}
& M_{\Sigma}=M_{0 x}(t)+M_{0 y}(t)+M_{0 z}(t)=F_{0}\left(e_{z} \cos \omega_{1} t-e_{y} \sin \omega_{1} t\right)+ \\
& +F_{0} e_{x} \cos \theta \cos \omega_{1} t+F_{0} e_{z} \cos \theta \sin \omega_{1} t= \\
& =F_{0} l_{m} \cos \theta\left[\sin \theta \cos \omega_{1} t-\operatorname{tg} \theta \sin ^{2} \omega_{1} t+\cos \theta \cos \omega_{1} t+\sin \theta \sin \omega_{1} t\right]= \\
& =F_{0} l_{m} \cos \theta\left[\sin \left(\theta-\omega_{1} t\right)+\cos \left(\theta-\omega_{1} t\right)-\cos \theta \sin \omega_{1} t\left(1+\frac{\sin \theta \sin \omega_{1} t}{\cos ^{2} \theta}\right)\right] .
\end{aligned}
$$

\subsection{Vibrations in the precession reducer with a vertical plane of symmetry}

Regarding simplified scheme of the dynamic PPT model (Figure 2), the dynamic model is characterized by three degrees of freedom $y, z, \varphi$ corresponding to movements $\left(y, \varphi_{x}\right)$ and vertical displacement $z$.

Differential equations of motion are:

$$
\left\{\begin{array}{c}
m \ddot{Y}+4 k_{x} Y-4 k_{y} a_{z} \varphi_{x}=F_{0} \cos \theta \sin \omega_{1} t \\
\dot{J}_{x} \ddot{\varphi}_{x}+4\left(k_{z} a_{y}^{2}+k_{y} a_{z}^{2}\right) \varphi_{x}-4 k_{y} a_{z} Y=F_{0} \sin \theta e \sin \left(\omega_{1} t-\alpha\right) \\
m \ddot{Z}+4 k_{z} Z=F_{0} \sin \theta \sin \omega_{1} t .
\end{array}\right.
$$

\subsubsection{Own vibrations}

For movements $\left(Y, \varphi_{x}\right)$, the vibration equations are: 


$$
\left\{\begin{array}{c}
m \ddot{Y}+4 k_{y} Y-4 k_{z} a_{z} \varphi_{x}=0 \\
\dot{J}_{x} \ddot{\varphi}_{x}+4\left(k_{z} a_{y}^{2}+k_{y} a_{z}^{2}\right) \varphi_{x}-4 k_{y} a_{z} Y=0 .
\end{array}\right.
$$

The solutions with following form $Y=Y_{0} \sin p t, \varphi_{x}=\varphi_{0} \sin p t$ and $\ddot{Y}=-p^{2} Y_{0} \sin p t, \ddot{\varphi}_{x}=-p^{2} \varphi_{0} \sin p t$, lead to the system of equations expressed by $Y_{0}$ and

$$
\begin{gathered}
\varphi_{0}:\left\{\begin{array}{c}
\left(-m p^{2}-\tilde{k}_{y}\right) Y_{0}-\tilde{k}_{x} a_{z} \varphi_{0}=0 ; \\
-\tilde{k}_{y} a_{z} Y_{0}+\left(-J_{x} p^{2}+\tilde{k}_{z} a_{y}^{2}+\tilde{k}_{y} a_{z}^{2}\right) \varphi_{0}=0,
\end{array}\right. \\
\text { Where: }\left\{\begin{array}{c}
\tilde{k}_{x}=\Sigma k_{y}=4 k_{y} ; \\
\tilde{k}_{z}=\Sigma k_{z}=4 k_{z} ; \\
\omega_{z}^{2}=\frac{k_{z}}{m} ;
\end{array} ;\left\{\begin{array}{c}
J_{x}=m \rho_{x}^{2}=\tilde{k} \frac{\rho_{x}^{2}}{\omega_{z}} ; \\
J_{x} m=\tilde{k}_{z}^{2} \frac{\rho_{x}^{2}}{\omega_{z}^{4}},
\end{array}\right.\right.
\end{gathered}
$$

and $\omega_{z}$ is the own pulsation of the precessional planetary reducer, $\rho_{x}=l_{m}-\sin \theta$ the radius of the body with mass $m$.

The compatibility condition is that the determinant of the equation system is null

$$
m J_{x} p^{4}-m\left(\tilde{k}_{z} a_{y}^{2}-\tilde{k}_{y} a_{z}^{2}\right) p^{2}-J_{x} \tilde{k}_{y} p^{2}+\tilde{k}_{y} \tilde{k}_{z} a_{y}^{2}=0
$$

\subsubsection{Forced vibrations}

The dynamic response of the precession reducer in the stabilized mode, for known $\omega_{l}$, is described with the expressions of vibration amplitudes as exciting pulse $\omega_{l}$ functions:

- amplitude of linear vibrations on the $y$ axis:

$$
A_{y}\left(\omega_{1}\right)=\frac{m_{0} r \omega_{1}^{2}}{\tilde{k}_{z}} \cdot \frac{B\left(\omega_{1}\right)}{D\left(\omega_{1}\right)},
$$

Where $B$ and $D$ are determined with the relations:

$$
B\left(\omega_{1}\right)=\sqrt{\left[\frac{\tilde{k}_{y}}{\tilde{k}_{z}} \cdot \frac{a_{z}}{\rho_{x}}\left(\frac{a_{z}}{\rho_{x}}-\frac{e_{z}}{\rho_{x}}\right)+\frac{a_{y}^{2}}{\rho_{x}^{2}}-\frac{\omega_{1}^{2}}{\omega_{z}^{2}}\right]^{2}+\left[\frac{\tilde{k}_{y}}{\tilde{k}_{z}} \cdot \frac{e_{y}}{\rho_{x}} \cdot \frac{a_{z}}{\rho_{x}}\right]^{2}}
$$

And :

$$
D\left(\omega_{1}\right)=\left[\frac{\omega_{1}}{\omega_{z}}\right]^{4}-\left[\frac{\omega_{1}}{\omega_{z}}\right]^{2}\left[\frac{a_{y}^{2}}{\rho_{x}^{2}}+\frac{\tilde{k}_{y}}{\tilde{k}_{z}} \cdot \frac{a_{z}^{2}}{\rho_{x}^{2}}+\frac{\tilde{k}_{y}}{\tilde{k}_{z}}\right]+\frac{\tilde{k}_{y}}{\tilde{k}_{z}} \cdot \frac{a_{y}^{2}}{\rho_{y}^{2}}
$$


- the magnitude of the angular vibrations around the $O x$ axis (pitching) determined with the relation:

$$
A_{\varphi}\left(\omega_{1}\right)=\frac{m_{0} r \omega_{1}^{2} C\left(\omega_{1}\right)}{\tilde{k}_{z} \cdot \rho_{x} \cdot D\left(\omega_{1}\right)},
$$

Where $\mathrm{C}$ is determined by the relationship

$$
C\left(\omega_{1}\right)=\sqrt{\left[\frac{\tilde{k}_{y}}{\tilde{k}_{z}}\left(\frac{a_{z}}{\rho_{x}}-\frac{e_{z}}{\rho_{x}}\right)+\frac{e_{z}}{\rho_{x}} \cdot \frac{\omega_{1}^{2}}{\omega_{z}^{2}}\right]^{2}+\left[\frac{e_{y}}{\rho_{x}}\left(\frac{\tilde{k}_{y}}{\tilde{k}_{z}}-\frac{\omega_{1}^{2}}{\omega_{z}^{2}}\right)\right]}
$$

- the amplitude of linear vibrations along the $O z$ axis:

$$
A_{z}=\frac{m_{0} r \omega_{1}^{2}}{\tilde{k}_{z}} \cdot \frac{1}{1-\frac{\omega_{1}^{2}}{\omega_{z}^{2}}}
$$

\section{Identifying resonant frequencies}

To analyze the vibrations of precessional planetary transmissions, which are actually more or less complex mechanical systems, the energy and vibration sources on which this energy is propagated must be taken into account. Energy is always transferred as energy flows from the vibration source to the energy absorber where it is converted to heat. In some cases, the propagation path may be very short, but in others the energy flow can be propagated over relatively long distances before it is absorbed.

An oscillating mechanical system can be excited by harmonic, periodic or random forces. The oscillating mechanical system vibrates in a certain way, at each of these oscillations its movement can be characterized by a given number of parameters, the determination of the values of which means finding out the response of the whole system to the excitation to which it is subjected. The response will depend on the characteristics of the oscillating system and the excitation parameters.

Resonance occurs when the frequency of an excitation force applied to a system coincides with the system's own frequency, causing a great amplification of system vibrations. This gain depends on system damping.

Own frequencies can be excited by a harmonic oscillation if it has enough energy to excite the system and if its frequency has a value around its own frequency.

The resonance phenomenon is recognized by the large amplitude variation and the phase angle change at low speed variations, which depend on system damping and can be determined in several ways:

- by calculation using modal analysis by computer programs, where the resonance frequencies obtained are not accurate and their values are very close to the real ones;

- by practical test with a hammer, when the mechanical structure is struck and at the same time the impact response of the system is measured, followed by the analysis of the obtained data, which usually results in the first resonance frequency; 
- by actual measurements, by analyzing vibration data measured during start-up or shutdown of the machine being studied, in stationary operation at a speed higher than the speed or critical speeds.

\section{Constructive-technological solutions for noise and vibration reduction}

Practically in vibro-acoustical diagnostics $[8,9]$ there are various methods to minimize the vibration and noise level in the technical and energetic machines and devices. In the other way the mechanical transmissions used in various machines, installations are sources of vibration and high frequency noise. The most efficient but also the most expensive method for obtaining silent transmissions is the method of processing the parts with very high precision or the static and dynamic balancing of the moving parts.

As conclusion most simpler and cheapest is constructivist-technological methods such as:

a) Manufacture of a plastic gear or sintered metallic powder wheel [ 5,7];

b) Compensation of manufacturing and assembly errors [ 3,10];

c) Use of intermediate elastic thin- layered rubber metal located between the contact surfaces of the gear [6];

\section{References}

1. Iu. Malcoci, Vibro acoustic research of planetary precessional transm., Doctoral thesis, UTM, 135 (2015)

2. I. Bostan, V. Dulgheru, A. Sochirean, Precessional planetary transmission (in romanian), Patent MD-2821 (2005)

3. I. Bostan, V. Dulgheru, Iu. Malcoci, Precessional planetary transmission, (in romanian) Patent MD-4354 (2015)

4. V. Dulgheru, Statical and dynamic precessional planetary transmission. $\mathrm{PhD}$. Thesis, 350 (1995).

5. Iu. Malcoci, I. Bodnariuc, Vibroacoustical diagnosis of planetary precessional kinematical transmission (Part 2), ACME, Iaşi, 4 (2016)

6. I. Dicusară, Iu. Malcoci, Planetary Precession Gear with Thin-Layered Ruber Metal, Non. Tech. Review 2, 4, (2016)

7. Iu. Malcoci, I. Bodnariuc, Vibroacoustical diagnosis of planetary precessional kinematical transmission (Part 1), IManE 2015. Published in: Applied Mechanics and Materials Vols. 809-810, (2015)

8. Iu. Malcoci, , Meridian Ingineresc 3, (2014).

9. Iu. Malcoci, Meridian Ingineresc 3, (2013)

10. Iu. Malcoci, Trans Tech Publ. Ltd, Switzeland, (2014) 